\title{
A Review on Cybersickness and Usability in Virtual Environments
}

\author{
Maryam Mousavi ${ }^{a}$, Yap Hwa Jen ${ }^{b}$ and Siti Nurmaya Binti Musa ${ }^{c}$ \\ Department of Engineering Design and Manufacture, Faculty of Engineering, University of Malaya, \\ 50603 Kuala Lumpur, Malaysia. \\ amousavi.maryam@gmail.com, bhjyap737@um.edu.my, 'nurmaya@um.edu.my
}

Keywords: Cybersickness, Usability, Virtual reality, Virtual environment.

\begin{abstract}
With the emerge of new technologies many systems are presented to a wider range of users at reasonable costs. Virtual Reality (VR) technology has also entered many new economical areas such as tourism, business, online games, and also cultural heritage. The new advancement in VR and its availability to the end user in many forms necessitates considering the health issues because cybersickness is one of the drawbacks of Virtual Environments (VE). In addition, usability of the VE and the provided VR technology and system is of paramount importance in the market to attract the user. However, usability measurement of the VE also has become a difficult issue due to the vast range of products and users. A review on the cybersickness and usability issues in VE is prepared and presented in this paper.
\end{abstract}

\section{Introduction}

In this era of science and technology, improvements and developments in every field are rapidly taking place. Virtual Reality (VR) technology with its wide range of influence on industry and research is respectively developing. However, despite all the improvements made, some problems are yet to be overcome. Cybersickness and usability are two of the characteristics that need to be improved in the VR area of research. This paper looks into the issues causing the cybersickness and its effect reduction and control methods. The usability characteristic in virtual environments is also reviewed from different aspects.

\section{Cybersickness}

Considering health and safety issues in virtual environment technology, cybersickness is one of the important aspects to deal with. Cybersickness (CS) is a form of motion sickness that occurs to people who experience VEs. Cybersickness poses a serious threat to the usability of VE systems. Different levels of sickness (from headaches to severe nausea) may happen to people who use VE systems $[1,2]$.

Cause of cybersickness. Three main theories are known as the causes of cybersickness: (1) the sensory conflict theory, (2) the postural instability theory, and (3) the poison theory. The first theory is the oldest and the most accepted theory relating to motion sickness and cybersickness [3]. This theory explains that conflicts between visual and vestibular systems are the main cause for cybersickness when the subject does not get the expected response, a conflict occurs and cybersickness may ensue. Riccio and Stoffregen [4] developed the postural instability theory, which is based on maintaining postural stability in the environment. In this case, postural stability is when its uncontrolled movements of the perception and action systems are minimized. The poison theory tries to describe why motion sickness and cybersickness happen from an evolutionary standpoint [5]. The theory suggests that the ingestion of poison causes physiological effects involving the coordination of the visual, vestibular, and other sensory input system. Sometimes adverse simulation may influence the visual and vestibular system in such a way that make misunderstanding for the body that thinks it has ingested some type of toxic substance thus causing disturbing symptoms which lead to an emetic response. It is discussed that the cause of 
cybersickness is based on a maladaptive process which originally used to help the body get rid of toxic substances [6].

There are a number of factors that have shown considerable contribution to cybersickness in virtual environments that do not fall into any of the predefined theories [7, 8]. Display and technology issues, position tracking error, lag, and flicker issue are some of the unclassified factors causing cybersickness. Other than the causing factors, it is interesting to study the human body response to this event. Based on the human body mechanism complexity,certain situations may have different effects on different people. To investigate this complexity, some factors have been studied to address this matter.

1- Gender: women are more susceptible to cybersickness than men [9]. Because they have wider fields of view than men and it increases the chance of flicker sensitivity [7].

2- Age: age plays a critical role in cybersickness susceptibility. It is argued that severe cybersickness happens between the ages of 2 and 12 years, and then it decreases till the age of 21 and more slowly thereafter till reaching the level nonexistent at the age of 50 [3].

3- Illness: If someone has an illness, he/she may experience different cybersickness. Position in the simulator: Users of VEs may experience different cybersickness, based on Based on their position in the simulator.

Cybersickness Reduction. cybersickness is a serious drawback in application of VEs and it should either be eliminated completely or at least reduce its severity. Some of the methods that have been used to reduce the cybersickness symptoms are as below:

1. Motion platforms: Based on the sensory conflict theory, if sickness happens in a VE, it is a sign which implies a conflict between the visual and vestibular systems. Thus, to reduce the cybersickness the idea of adding motion platforms to the VE simulator was introduced by Casali [10].

2. Direct vestibular stimulation: Application of direct vestibular stimulation can also reduce the cybersickness. A nearly similar concept as motion platform is proposed in this method, but the large motion base is altered by wearing a device which transfers electrical signals to the 8th cranial nerve. Signals then trick the vestibular system into sensing that a linear or angular acceleration and deceleration is taking place [10].

3. Rest frames: The rest frame idea is based on the strong human insight of stationary surface and stuff [11]. Accordingly, by providing a rest frame in the VE the user can use it for stationary and reduce and avoid the cybersickness.

4. Adaptation: The more credited approach is to plan an adoption program for the VE users to prepare for VE [12].

Having discussed the age matter in cybersickness, Liu and Uang [13] found that the Simulator Sickness Questionnaire (SSQ) scores of cybersickness increased significantly with increasing navigation rotating speed and exposure duration on older participants. The device of thin-film transistor liquid-crystal display (TFT-LCD) has been utilized to present the VE to the participants. Therefore, the cybersickness-predicting model was designed to evaluate the symptom of cybersickness for older users on TFT-LCD display in VE.

Kennedy and Stanney [14] found that sometimes postadaptation phenomena can happen even when there is no sickness. They discussed that it is possible that any of the aftereffects happen without sickness. However, sickness in VEs is a problem which is depending on people and different effects may occur in different individuals [15]. One of the researches that could make a successful pace in cybersickness control was done by Sharples et al. [16] many other researchers have continued to find a system where can reduce or avoid the sickness in VE. Stanley and Hash [17] have done an experiment on 24 student participants that they were immersed in a VE. They tested the effect of user-initiated control on changing the level of cybersickness and reported high level of control by each participant in the VE.

It is discussed that symptoms of simulator sickness are the same as motion sickness and studies outcome of motion sickness can be used in cybersickness studies. On the contrary, it is reported that 
observing numerous visitors to VE reveals the inconsistency between the traditional understanding of motion sickness and cybersickness, where in cybersickness elderies are more prone to get sick in VE compared to youngsters and this is vice versa in motion sickness. Therefore, traditional concept of motion sickness and especially its relationship with age may not be true in the case of cybersickness [18].

\section{Usability}

Usability is one of the crucial characteristics in human computer interaction. The term Usability substituted the User Friendliness term in computer based technologies [19]. Based on different approaches to usability measurement, there are different definitions of usability. In VR technology the efficiency of the system is measured according to the usability measurement features. A virtual reality interface with high usability provides the user with complete accessibility and freedom in managing and accomplishing his/her task in the VE [20].

Usability Evaluation. Sweeney et al. [21] is the first researcher that defined usability as "the capability in human functional terms to be used easily and effectively by the specified range of users, given specified training and user support, to fulfill the specified range of tasks, within the specified range of environmental scenarios". Proposed four dimensions to measure usability:

1. Effectiveness: performance in task completion.

2. Learnability: degree of learning to accomplish tasks.

3. Flexibility: adapt to variation in tasks.

4. Attitude: user satisfaction with the system [21].

Among different definitions of usability, Bevan [19] defined it as "quality in use" and introduced three criteria for measuring usability which are understandablity, learnability, and operability. Nielsen [22] rephrased the usability term definition given by Sweeney et al. [21] and stated that usability is a feature that influences product acceptance. He mentioned that the measurement of usefulness should be assessed individually and five characters are introduced to be used as follows:

1-Learnability: the system should be easy to learn;

2-Efficiency: the system should be efficient to use;

3-memorability: the system should be easy to remember;

4-low error rate: the system should make users enable to make fewer errors when they are using the system

5-satisfaction: Users should be satisfied with the system.

In order to have standards for usability measurement, ISO 9241-11 [23] standards made guidelines for software interfaces. Based on ISO 9241-11 the elements of usability were, (1) effectiveness, (2) accuracy and (3) completeness. In addition, ISO 9126 [24] defined usability as a relatively independent contribution to software quality associated with the design and evaluation of the user interface and interaction. ISO 9126 [24] stated usability should be measured by the following features: (1) Understandability: the capability of the software product to enable the user to understand whether the software is suitable;

(2) Learnability: the capability of the software product to enable the user to learn its application; (3) Operability: the capability of the software product to enable the user to operate and control it; and

(4) Attractiveness: the capability of the software product to be attractive.

To prevent and reduce the common usability problems in VEs, interface design guidance is introduced as a helpful approach [25]. This approach is argued to be effective in terms of increasing the user spatial awarness in VEs and interaction and controlling objects in VE. It also stated that interface design guidance can enhance the sense of places by direction, linkage and atmosphere. To design a VE, Wann and Williams [26], highlighted the need of spatial elements such as places, paths, domains, and thresholds. They stated a well designed VR system should support perception, navigation, exploration, and engagement. 
Navigation and exploration of VEs are two of the usability problems that can severely affect VR systems. The lack of navigation cues to direct the users to the object and around VE, and navigating too close to virtual objects are argued to be the major causes of navigation and exploration issues in VEs [27]. It was earlier discussed by Kaur [25] that typical usability problems relate to the poor performances originated from the poor navigation design, unsuitable screen design, bad feedback, and lack of consistency for instance.

Some of the obstacles in defining the participants' performance within VEs are the wide range of interfaces to be used and various reactions and behaviors that might be exhibited within the interaction. Therefore, it is difficult to provide coherent guidance for designers [28]. Studies involving human factors are highly difficult to plan and carry out due to the numerous variables to be controlled and the costs involved in such study [29]. However, due to the importance of usability improvement in VEs which helps to reach the full potential of the technology, the need of guidelines and background information for alternative solution is highlighted [30].

\section{Conclusion}

The cybersickness and usability are two important issues which affect the efficacy of virtual environments. There are three theories that explain the cybersickness causes; (1) the sensory conflict theory, (2) the postural instability theory, and (3) the poison theory. There are also some other causness which are not directly related to the above theory such as, Display and technology issues, position tracking error, lag, and flicker issue. From an individual standpoint, age, gender, illness, and the position in the simulator are factors that interfering with severity of cybersickness. Aside from the cybersickness effects on the VE users, usability is a term which encompasses all the measurements to evaluate the efficiency of a system and human computer interaction in general. Usability is defined in different ways by different factors for usability evaluation. However, to summarize the influential factors in measuring and defining the usability of a system, understandability, learnability, operability, attractiveness, effectiveness, accuracy and completeness of a systems are introduced to be major factors. Overall, a virtual reality interface with high usability provides the user with complete accessibility and freedom in managing and accomplishing his/her task in the VE.

\section{Acknowledgment}

The authors would like to acknowledge the Ministry of Higher Education (MOHE) Malaysia for the financial support under High Impact Research Grant UM.C/HIR/MOHE/ENG/35 (D00003516001).

\section{References}

[1] R.S. Kalawsky, The Science of Virtual Reality and Virtual Environments. Addison-Wesley, Wokingham, England, 1993.

[2] K. Stanney, "Realizing the full potential of virtual reality: human factors issues that could stand in the way," Proceedings Virtual Reality Annual International Symposium '95, pp. 28-34, 1995.

[3] J. Reason, and J. J. Brand, Motion sickness / J. T. Reason, J. J. Brand Academic Press, London ; New York, 1975.

[4] G.E. Riccio and T.A. Stoffregen. An Ecological Theory of Motion Sickness and Postural Instability. Ecological Psychology, 3(3): 195-240, 1991.

[5] M. Treisman. Motion Sickness: An Evolutionary Hypothesis. Science, 197, 493-495, 1977.

[6] J.J. LaViola Jr. A Discussion of Cybersickness in Virtual Environments, 32(1): 47-56, 2000. 
[7] E.M. Kolasinski, S.L. Goldberg, and J.H. Miller. Simulator Sickness in Virtual Environments. Technical Report 1027, U.S. Army Research Institute for the Behavioral and Social Sciences, 1995.

[8] R. Pausch, T. Crea, and M. Conway. A Literature Survey for Virtual Environments: Military Flight Simulators Visual Systems and Simulator Sickness. Presence 1(3): 344-363, 1992.

[9] F. Biocca. Will Simulation Sickness Slow Down the Diffusion of Virtual Environment Technology? Presence 1(3): 334-343, 1992.

[10] J.G. Casali. Vehicular Stimulation-Induced Sickness, Volume 1 :An Overview. IEBOR Technical Report No. 8501. (NTSC TR 86-010), Orlando, FL:Naval Training Sys- tems Center, 1986.

[11] J.D. Prothero. ' 'The Role of Rest Frames in Vection, Presence and Motion Sickness", Ph.D Thesis, University of Washington, 1998.

[12] M.E. McCauley and T. J. Sharkey. Cyber- Sickness: Perception of Self-Motion in Virtual Environments. Presence, 1(3):311-318, 1992.

[13] C. Liu and S. Uang, "Measurement and Prediction of Cybersickness on,” pp. 666-675, 2007.

[14] R.S. Kennedy and K.M. Stanney. Postural instability induced by virtual realityexposure: Development of a certification protocol. International Journal of Human-Computer Interaction, $8(1), 25-47,1996$.

[15] R.S. Kennedy, J.M. Drexler, D.E. Compton, K.M. Stanny, D.S. Lanham, and D.L. Harm, "Configural Scoring of Simulator Sickness, Cybersickness and Space Adaptation Syndrome: Similarities and Differences?" In Virtual and Adaptive Environments: Applications, Implications, and Human Performance Issues (pp. 247-278), 2003.

[16] S. Sharples, S. Cobb, A. Moody, and J.R. Wilson, "Virtual reality induced symptoms and effects (VRISE): Comparison of head mounted display (HMD), desktop and projection display systems," Displays, vol. 29, no. 2, pp. 58-69, 2008.

[17] K.M. Stanney, P.A.K. Hash, Locus of user-initiated control in virtual environments: influences on cybersickness, Presence: Teleoperators and Virtual Environments 7 (5):447-459, 1998.

[18] L. L. Arns and M. M. Cerney, "The Relationship Between Age and Incidence of Cybersickness Among Immersive Environment Users,” vol. 2005, pp. 267-268, 2005.

[19] N. Bevan, Standards relevant to European Directives for display terminals. In: Bullinger (1991).

[20] I. Kossyk, D. Jonas, L. Raschend, and K. Kondak, "Usability of a virtual reality system based on a wearable haptic interface," pp. 3474-3479, 2011.

[21] M. Sweeney, M. Maguire, and B. Shackel, "Evaluating user-computer interaction: A framework.” In International Journal of Man-Machine Studies, 38, pp. 689-711. 1993.

[22] J. Nielsen. Usability engineering. Academic Press, Bos- ton, Massachusetts. 1993.

[23] ISO (1991a) Ergonomic requirements for office work with visual display terminals, ISO 9241.

[24] ISO (1991b) Software product evaluation - Quality characteristics and guidelines for their use, ISO DIS 9126.

[25] K. Kaur. Designing Virtual Environments for Usability, in INTERACT 97 (IFIP), S. Howard, J. Hammond and G. Lindgaard (eds.), Sydney, Chapman and Hall, 1997. 
[26] J.Wann , M. M. Williams. What does virtual reality need? Human factors issues in the design of three-dimensional computer environments. International Journal of Human-Computer Studies 44 (6), 829-847(19), 1996.

[27] S. Smith, T. Marsh, D. Duke, P. Wright. Drowning in immersion. In: Proceedings of UKVRSIG'98, Exeter, UK, 1998.

[28] J. Wilson, Interaction with Virtual environments. Int. J. Human-Computer Studies, 64, pp.157, $2006 a$.

[29] J. Wilson, M. D'Cruz. Virtual and interactive environments for work of the future. Int. J. Human-Computer Studies, vol. 64, pp158-169, 2006b.

[30] B.S. Santos, P. Dias, P. Santos, S. Silva, and C. Ferreira, "Usability evaluation in Virtual Environments through empirical studies involving users," ACM-CHI 2009 Workshop Challenges in Evaluating Usability and User Experience in Reality Based Interaction, Boston, USA, 2009. 